EPJ Web of Conferences 73, 03015 (2014)

DOI: $10.1051 /$ epjconf/20147303015

(C) Owned by the authors, published by EDP Sciences, 2014

\title{
Physics and outlook for rare, all-neutral Eta decays
}

\author{
David J. Mack ${ }^{\mathrm{a}}$ \\ TJNAF, 12000 Jefferson Avenue, Newport News, VA 23606, USA
}

\begin{abstract}
The $\eta$ meson provides a laboratory to study isospin violation and search for new flavor-conserving sources of $\mathrm{C}$ and $\mathrm{CP}$ violation with a sensitivity approaching $10^{-6}$ of the isospin-conserving strong amplitude. Some of the most interesting rare $\eta$ decays are the neutral modes, yet the effective loss of photons from the relatively common decay $\eta \rightarrow 3 \pi^{0} \rightarrow 6 \gamma(33 \%)$ has largely obscured rare decays producing 3-5y's. Particularly important relevant branches include the highly suppressed $\eta \rightarrow \pi^{0} 2 \gamma \rightarrow 4 \gamma$, which provides a rare window on testing models of $O\left(p^{6}\right)$ contributions in ChPTh, and $\eta \rightarrow 3 \gamma$ and $\eta \rightarrow$ $2 \pi^{0} \gamma \rightarrow 5 \gamma$ which provide direct constraints on $\mathrm{C}$ violation in flavor-conserving processes. The substitution of lead tungstate in the forward calorimeter of the GluEx setup in Jefferson Lab's new Hall D would allow dramatically improved measurements. The main niche of this facility, which we call the JLab Eta Factory (JEF), would be $\eta$ decay neutral modes. However, this could likely be expanded to rare $\eta^{\prime}(958)$ decays for low energy QCD studies as well as $\eta$ decays involving muons for new physics searches.
\end{abstract}

\section{Introduction}

The $\eta$ meson provides a laboratory to study isospin violation and search for new flavor-conserving sources of $\mathrm{C}$ and $\mathrm{CP}$ violation. [1] Because the $\eta$ has an unusually small decay width $\left(\Gamma_{t o t}=1.3 \mathrm{keV}\right)$ [3], a branching ratio upper limit of $O\left(10^{-6}\right)$ corresponds to a partial width 11 orders of magnitude smaller than for example the $\omega$ total decay width. The $\eta$ is perhaps the only hadron where it appears feasible to probe non-weak decays within an order of magnitude or so of the weak scale. While much progress has been made recently in the rare, charged decay modes (see section 3), progress in the rare, neutral modes has languished mainly due to a missing photon background from the relatively common $\eta$ decay mode $\eta \rightarrow 3 \pi^{0} \rightarrow 6 \gamma(33 \%)$. The problem is that when photons from this dominant branch are lost out of the acceptance (or showers merge in the calorimeter), then rare decays to 3-5 $\gamma$ 's can be overwhelmed by background.

To emphasize the importance of reducing background, we derive the figure of merit for a dataset. For the SM forbidden decays, the sensitivity of the branching ratio (henceforth BR) upper limit is limited by background $\left(N_{b k g}\right)$ fluctuations in the signal window. Thus in the no-signal scenario, at $95 \%$ confidence level the $B R<2 \sqrt{N_{b k g}} /\left(N_{\eta} \epsilon\right)$ where $N_{\eta}$ is the number of $\eta$ 's produced and $\epsilon$ is the acceptance $\mathrm{x}$ efficiency. Defining the luminosity-independent background fraction,

\footnotetext{
ae-mail: mack@jlab.org
}

This is an Open Access article distributed under the terms of the Creative Commons Attribution License 4.0, which permits unrestricted use, distribution, and reproduction in any medium, provided the original work is properly cited. 

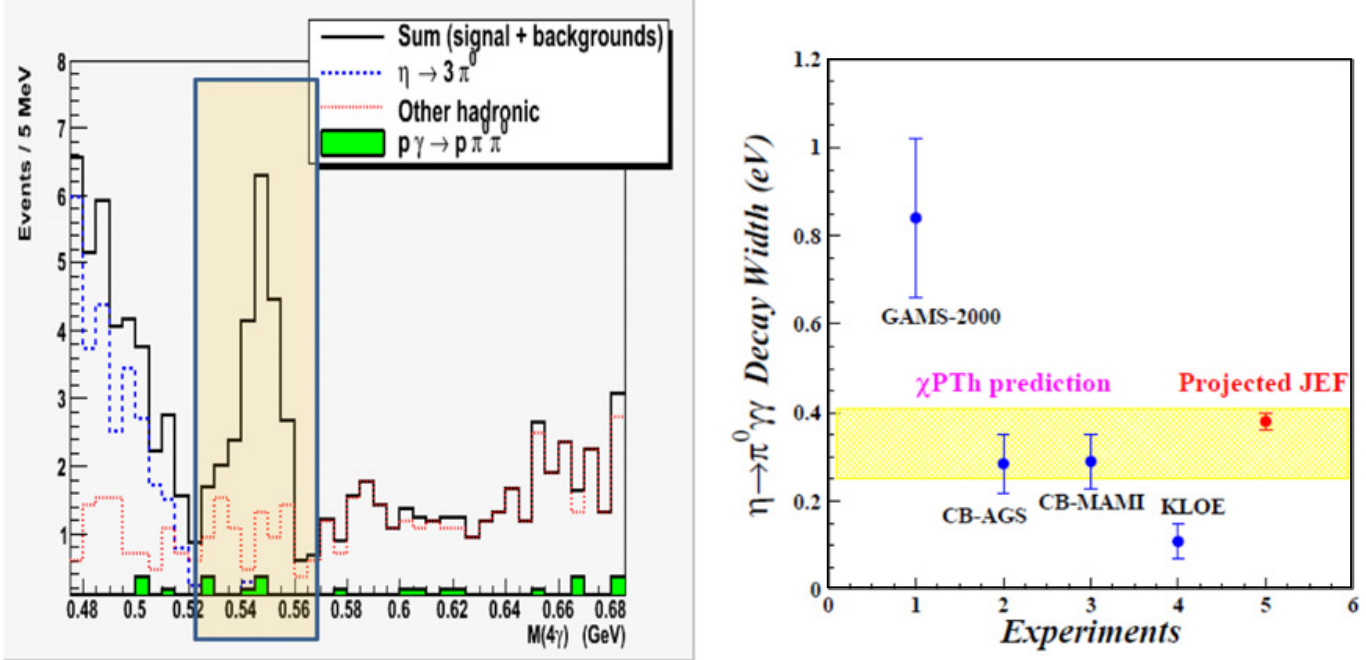

Figure 1. Left: simulated yield from 1 day in $M_{4 \gamma}$ histogram for an $\eta \rightarrow \pi^{0} 2 \gamma$ signal plus backgrounds at JEF after cuts applied. To the left of the signal box is the dangerous missing photon background. Right: previous experimental results for $\eta \rightarrow \pi^{0} 2 \gamma$ and a projection for JEF.

$f_{b k g} \equiv N_{b k g} /\left(N_{\eta} \epsilon\right)$, we obtain

$$
B R<2 \sqrt{f_{b k g} /\left(N_{\eta} \epsilon\right)}
$$

The appropriate figure of merit (FOM) to maximize is therefore $F O M \equiv N_{\eta} \epsilon / f_{b k g}$. Hence minimizing $f_{b k g}$ is as important as maximizing $N_{\eta} \epsilon$. Note that an order of magnitude reduction in a BR upper limit requires an improvement in FOM of 100! Rare decays are a tough game.

The addition of a high resolution lead tungstate core to the GluEx forward lead-glass calorimeter would allow dramatically improved neutral mode measurements (a reduction of 1-1.5 orders of magnitude in the BR). [2] Fast $\eta$ 's would be produced via $\gamma+p \rightarrow \eta+p$ using incident 9-11.7 GeV tagged photons along with recoil proton detection. Our name for the proposed facility is the JLab Eta Factory (JEF). The boost provides several advantages such as a reduced likelihood of losing a photon below threshold, and a greater likelihood that events with a lost photon will be removed by the missing energy cut. The missing energy cut also prevents feed-down from higher invariant masses into the $\eta$ invariant mass window, while a cut on co-planarity between the $\eta$ and recoil proton suppresses multistep decays like $\gamma+p \rightarrow \pi^{0}+X^{+} \rightarrow 2 \pi^{0}+p$.

Figure 1 (left) shows a PYTHIA projection for a single day of JEF data for the rare decay $\eta \rightarrow \pi^{0} 2 \gamma$. A small peaking background due $\eta \rightarrow 3 \pi^{0}$ with merged showers exists but is not evident in this low statistics simulation. JEF would represent a revolutionary improvement in signal/background.

\section{Physics interest in rare all-neutral Eta decays}

\subsection{Probing a highly suppressed meson decay at $O\left(p^{6}\right)$ in chiral perturbation theory}

After the two largest neutral $\eta$ decay modes, the doubly radiative decay $\eta \rightarrow \pi^{0} 2 \gamma$ is rarer by 3 orders of magnitude. Because even the dominant $\eta$ decay branches are highly suppressed, the partial decay width of $\eta \rightarrow \pi^{0} 2 \gamma$ is only $0.3 \mathrm{eV}$. [3] In terms of ChPTh, this reaction is intriguing because the first sizable contributions occur at $O\left(p^{6}\right)$ [4] hence measurements provide a rare opportunity to test models for such relatively high order. [5] Contributions from scalar mesons to the overall BR appear to be small; the 
Table 1. Matrix of $\mathrm{C}$ and $\mathrm{P}$ symmetries. Assuming invariance under CPT, then interactions fall into one of only four combinations. The off-diagonal combinations involve CP violation, with EDM's most important for constraining new C,PV,CPV interactions, while $\eta$ decays directly constrain new CV,P,CPV interactions.

\begin{tabular}{|c|c|c|}
\hline & $\mathrm{P}$ & PV \\
\hline $\mathrm{C}$ & $\mathrm{C}, \mathrm{P}, \mathrm{CP}$ & $\mathrm{C}, \mathrm{PV}, \mathrm{CPV}$ \\
& Strong, EM & Weak (at 2-loop level) \\
& $\eta \rightarrow 2 \gamma$, & EDMs, \\
& $\eta \rightarrow 3 \pi^{0}$, etc. & $\eta \rightarrow 2 \pi$ \\
\hline $\mathrm{CV}$ & $\mathrm{CV}, \mathrm{P}, \mathrm{CPV}$ & $\mathrm{CV}, \mathrm{PV}, \mathrm{CP}$ \\
& Weak (at 2 -loop level) & Weak \\
& $\eta \rightarrow 3 \gamma$, & PV experiments, \\
& $\eta \rightarrow 2 \pi^{0} \gamma$, etc. & $\mu$ and $\beta$ decay asymmetries \\
\hline
\end{tabular}

Dalitz distribution $d \Gamma / d m_{\gamma \gamma}$ will demonstrate this more clearly. [6] Precise measurements of $\eta \rightarrow \pi^{0} 2 \gamma$ may also help reduce uncertainties in long distance CP-conserving contributions to $K_{L} \rightarrow l^{+} l^{-}$[7].

Figure 1 (right) shows published and preliminary results for the $\eta \rightarrow 2 \gamma$ BR along with our projected uncertainty of $<5 \%$. The current experimental situation is arguably ambiguous at the factor of 2 level, almost certainly due to poor Signal/Background in the existing experiments. The $d \Gamma / d m_{\gamma \gamma}$ distribution contains much more information than the BR and is the critical observable; the BR is merely a quick way to graphically portray the experimental situation.

\subsection{Physics beyond the standard model}

One approach to searching for physics beyond the SM is to search for violations of symmetries which are largely respected in the SM. Assuming CPT is a good symmetry, Table 1 classifies forces in the SM according to their properties under charge conjugation $(\mathrm{C})$ and parity $(\mathrm{P})$.

While the strong and electromagnetic interactions are thought to conserve $\mathrm{C}$ and $\mathrm{P}$, in the weak interaction both $\mathrm{C}$ and $\mathrm{P}$ are violated (lower right quadrant in Table 1). Since highly precise parity violating asymmetry experiments on the $e, p$, and atomic Cs are consistent with the SM, any new interaction in this quadrant affecting the first generation of quarks and leptons cannot exceed a few $\%$ of the weak amplitude.

In the upper right quadrant of Table 1 , we encounter $\mathrm{CP}$ violation in which $\mathrm{C}$ is conserved but $\mathrm{P}$ is violated. The SM background here for flavor-conserving processes is extremely small since it occurs at two-loop level. However, larger contributions for EDMs could in principle arise at one-loop level from SUSY. For $\eta \rightarrow 2 \pi$, calculations with an extended Higgs sector found 12 orders of magnitude enhancement in the C,PV,CPV decay compared to the base SM prediction, but the predicted BR of $O\left(10^{-15}\right)[8]$ is still far below the sensitivity of any forseeable experiment. Nevertheless, other beyond SM calculations may predict bigger BRs, for example by invoking the large $s \bar{s}$ content of the $\eta$. [9] We will lower the BR upper limit for $\eta \rightarrow 2 \pi^{0}$ by over an order of magnitude.

A less constrained possibility is that of $\mathrm{C}$ violation with $\mathrm{P}$ conservation (the lower left quadrant in Table 1). Under CPT this is of course equivalent to $\mathrm{T}$ violation with $\mathrm{P}$ conservation. There are few ways to place direct constraints on C,P,CP interactions, and none are apparently more sensitive than $\eta$ decays. Of relevance to JEF are $\eta \rightarrow 2 \pi^{0} \gamma$ (isoscalar) and $\eta \rightarrow 3 \pi^{0} \gamma$ (isovector) [10], as well as $\eta \rightarrow 3 \gamma$. The magnitude of such a new interaction is indirectly constrained by EDM measurements since a CV,P,CPV interaction could mix with the usual weak interaction to yield C,PV,CPV. However, these indirect constraints from EDMs turn out to be hierarchically ambiguous [11], so direct constraints on $\mathrm{C}$ violation with $\mathrm{P}$ conservation with sensitivity as close as possible to the weak scale would be very interesting. 


\section{Other potential applications of the JEF facility}

JEF's critical role in rare $\eta$ decay neutral modes is clear, but is less obvious for charged final states where there has been tremendous recent progress relevant to muon g-2 [12], searches for a dark photon and a new pseudo-scalar particle [13], and 2nd class currents in the weak interaction [14] (to name just a few examples). If there is any low-hanging fruit remaining in the charged sector of rare $\eta$ decays, it is probably muon flavored. The planned addition of forward muon identification to Hall $\mathrm{D}$ by the Charge Pion Polarizability collaboration might allow the JEF to place improved constraints on lepton flavor violation in $\eta \rightarrow \mu^{+-} e^{-+}$, an extension of the 2 nd class current searches to $\eta \rightarrow \pi^{+} \mu^{-} \overline{\nu_{\mu}}+$ c.c., as well as constraints on the mass of a potential CP-odd light Higgs via $\eta \rightarrow \pi^{0} \mu^{+} \mu^{-}$.

The JEF niche of rare, neutral $\eta$ decays should be extendable to other not-so-light neutral mesons which produce large photon backgrounds. For example, the $\eta^{\prime}(958)$ has a large branch, $\eta^{\prime} \rightarrow 2 \pi^{0} \eta$ (22\%), which not only obscures the two rare decays $\eta^{\prime} \rightarrow \pi^{0} 2 \gamma$ and $\rightarrow \eta 2 \gamma$ (the latter with significant scalar meson contributions [6]) but $\eta^{\prime} \rightarrow 3 \pi^{0}$ [15] which helps determine $\left(\left(m_{d}-m_{u}\right) / m_{s}\right)^{2}$ [16]. In a case where the final state contains a mixture of charged and neutral pions, the large branch $\eta^{\prime} \rightarrow \pi^{+} \pi^{-} \eta$ (43\%) has probably limited the sensitivity of searches for the rare decay $\eta^{\prime} \rightarrow \pi^{+} \pi^{-} 2 \pi^{0}$ which could provide another window into ChPTh at $O\left(p^{6}\right)$ [17].

I'd like to acknowledge the hard work of the JEF proposal writing committee and helpful comments from Elton Smith. Some opinions expressed are my own. This work was supported by DOE contract No. DE-AC0506OR23177, under which Jefferson Science Associates, LLC operates Thomas Jefferson National Accelerator Facility.

\section{References}

[1] S. Kullander et al., Acta Physica Polonica B 29, 97-111 (1998); A. Kupsc et al., J. Phys. Conf. Ser. 335, 012017 (2011), arXiv:1103.3860 [hep-ph]

[2] JLab proposal PR12-12-003, Symmetry Tests of Rare Eta Decays to All-Neutral Final States, May 4, 2012. http://www. jlab.org/exp_prog/proposals/12/PR12-12-003.pdf

[3] J. Beringer et al. (Particle Data Group), PR D 86, 010001 (2012) and 2013 partial update

[4] Ll. Ametller et al., Phys. Lett. B 276, 185-190 (1992)

[5] E. Oset et al., Phys. Rev. D 77, 073001 (2008), and ibid 67, 073013 (2003)

[6] R. Escribano, http://pos.sissa.it/archive/conferences/171/115/Conf inement X_115.pdf

[7] L.M. Sehgal, Phys. Rev. D 38, 808 (1988)

[8] C. Jarlskog et al., Phys. Rev. D 526327 (1995), and references

[9] C.Q. Geng et al., Modern Physics Letters A 17, 1489-1497 (2002)

[10] B.M. Nefkens et al., Phys. Rev. Lett. 94, 041601 (2005)

[11] A. Kurylov et al., Phys. Rev. D 63, 076007 (2001)

[12] P. Aguar-Bartolome et al., arXiv:1309.5648v1 [hep-ex] 22 Sep 2013

[13] G. Agakishiev et al., arXiv:1311.0216v1 [hep-ex] 1 Nov 2013

[14] M. Ablikim et al., Phys. Rev. D 87, 032006 (2013)

[15] M. Ablikim et al., Phys. Rev. Lett. 108, 182001 (2012)

[16] D.J. Gross et al., Phys. Rev. D 19, 2188-2196 (1979)

[17] A. Wirzba, Phys. Rev D 85, 014014 (2012) 